\title{
FAST SINGULAR BOUNDARY METHOD: MATHEMATICAL BACKGROUND AND APPLICATION IN WAVE PROPAGATION ANALYSIS
}

\author{
ZHUO-JIA FU ${ }^{1,2}$, JUN-PU LI $^{1} \&$ QIANG XI ${ }^{1}$ \\ ${ }^{1}$ Center for Numerical Simulation Software in Engineering and Sciences, Hohai University, China \\ ${ }^{2}$ Institute of Continuum Mechanics, Leibniz University Hannover, Germany
}

\begin{abstract}
Despite active research of many decades, numerical solution of the large-scale exterior wave problems remains a great challenge. The finite element method (FEM) needs to be coupled with some effective special treatments for handling unbounded domains, which are often tricky and largely based on trial-error experiences. The boundary element method (BEM) appears attractive to exterior problems, because the fundamental solutions satisfy the governing equation and Sommerfeld radiation condition at infinity. However, the BEM encounters computationally expensive singular numerical integration of fundamental solutions. To avoid this troublesome issue, the method of fundamental solutions (MFS) distributes the source nodes on a fictitious boundary outside the physical domain and is meshless, integration-free, and highly accurate. However, the placement of fictitious boundary is still an open issue for complex-shaped boundary and multiply connected domain problems. Recently, an alternative meshless boundary collocation approach, singular boundary method (SBM), has been proposed to solve various wave propagation problems. The key issue of the SBM is to determine the accurate source intensity factors instead of the singularities of fundamental solutions at origin. Several techniques have been proposed and investigated. The SBM is mathematically simple, easy-to-program, meshless, and applies the concept of source intensity factors to eliminating the singularity of the fundamental solutions. The method avoids singular numerical integrals in the BEM and circumvents the troublesome placement of the fictitious boundary in the MFS. This paper first presents the mathematical background of the SBM, and then applies it to wave propagation analysis.
\end{abstract}

Keywords: fundamental solutions, singular boundary method, boundary element method, wave propagation.

\section{INTRODUCTION}

Wave propagation has extensive and wide-range applications in engineering and sciences, especially in geological exploration, photovoltaic devices and metamaterials and ultrasonic non-destructive testing (NDT). The finite element method (FEM) and boundary element method (BEM) are two dominant numerical methods in the field of computational wave propagation. Since wave propagation analysis frequently refers to infinite domain problem, the domain-discretization FEM requires some additional efficient treatments to carefully truncate the infinite domain to finite domain, which are often tricky and largely based on trial-error experiences. It is natural to employ the BEM [1] in this kind of application without domain discretization since the semi-analytical fundamental solutions are used as basis functions, which satisfy the governing equation and the Sommerfeld radiation condition at infinity. Therefore, the BEM is an attractive alternative to the FEM especially for wave propagation under infinite domain. However, the BEM encounters sophisticated mathematical and computationally expensive numerical integration over the singularities.

To avoid this inconvenience in the BEM, the method of fundamental solutions (MFS) [2] places the source nodes on a fictitious boundary outside the physical domain, and implements a linear combination of fundamental solutions to approximate the numerical solution. In comparison with the BEM, the MFS provides more accurate solutions with the meshless and 
integration-free properties. However, the placement of source nodes on the fictitious boundary is vital for its numerical accuracy and stability, which is still an open issue especially for complex-shaped or multi-connected domains.

During the last two decades, great attentions have been paid to develop an accurate and stable semi-analytical boundary discretization scheme to eliminate the singularities of fundamental solutions in the BEM and also avoid the troublesome placement of fictitious boundary in the MFS. Generally speaking, there are two ways to achieve this goal. One is to derive nonsingular radial basis function general solutions instead of the singular fundamental solutions as basis function, for example, boundary knot method, boundary collocation method and modified collocation Trefftz method etc. The other one is to introduce the regularized technique to overcome the singularities of fundamental solutions, for example, regularized meshless method, modified method of fundamental solutions, singular boundary method (SBM) and boundary distributed source method etc.

This study focuses on the singular boundary method, which has been first proposed by Prof. Chen in 2009 [3]. Later, Fu and Chen [4] applied the SBM to wave propagation analysis. It inherits the merits from the BEM and the MFS and introduces the concept of the "origin intensity factors" or "source intensity factors" to eliminate the singularity in the fundamental solutions and instead of the numerical computation of the singular integrals in the BEM. There are several approaches [5]-[7] proposed to determine the origin intensity factors of both the fundamental solutions and their derivatives in the SBM application. The efficiency and accuracy of these approaches in the SBM have been verified for Laplace equations, Helmholtz equations, acoustic waves, elastic waves, and water waves with complicated-geometry domains.

This paper presents the fast singular boundary method for large-scale wave propagation application. The key issue is to overcome the ill-conditioning dense matrix generated from the SBM. So far, several fast dense matrix solvers have been employed, such as the Fast Fourier Transform (FFT) [7], the fast multipole methods (FMM) [8] dual-level scheme [9] and the multi-level scheme [10] etc.

A brief outline of the paper is as follows: in Section 2, a detailed description of the proposed fast SBM model is presented. Section 3 provides several numerical examples to demonstrate the effectiveness of the proposed method. Finally, in Section 4 some conclusions are drawn from the present analysis.

\section{FAST SINGULAR BOUNDARY METHOD FOR WAVE PROPAGATION}

Consider the time-harmonic wave propagating in a homogeneous medium $D$ exterior to an object bounded with the closed curve $\Gamma$, which can be described by Helmholtz equation:

$$
\nabla^{2} u(x)+k^{2} u(x)=0, \quad x \in D,
$$

subjected to the boundary conditions

$$
\begin{gathered}
u(x)=\bar{u} \quad x \in \Gamma_{D}, \\
q(x)=\frac{\partial u(x)}{\partial \mathbf{n}}=\bar{q} \quad x \in \Gamma_{N},
\end{gathered}
$$

where $u$ is the complex-valued amplitude of the radiated and/or scattered waves (velocity potential or wave pressure): 


$$
u= \begin{cases}u_{R}=u_{T}, & \text { if radiation, } \\ u_{S}=u_{T}-u_{I}, & \text { if scattering, } \\ u_{R+S}=u_{T}-u_{I}, & \text { if both }\end{cases}
$$

in which the subscripts $T, R, S, I$ denote the total, radiated, scattered and incident waves, respectively. $k=\omega / c$ is the wavenumber, $\omega$ the angular frequency, $c$ the wave speed in homogeneous medium $D$, and $\mathbf{n}$ the outward normal unit vector on the closed curve $\Gamma=\Gamma_{D}+\Gamma_{N}$, in which $\Gamma_{D}$ and $\Gamma_{N}$ represent the boundaries with the essential boundary (Dirichlet) conditions and the natural boundary (Neumann) conditions, respectively. For the exterior wave propagation problems, all scattered and radiated waves should be outgoing. This constraint condition is also known as the well-known Sommerfeld radiation condition:

$$
\lim _{r \rightarrow \infty} r^{\frac{1}{2}(\operatorname{dim}-1)}\left(\frac{\partial u}{\partial r}-i k u\right)=0
$$

where $\operatorname{dim}$ is the dimension of the acoustic problems, and $i=\sqrt{-1}$.

\subsection{Singular boundary method}

Similar to the MFS, the SBM approximate solutions $u(x)$ and $q(x)$ of exterior wave propagation problem (eqns (1) and (2)) can be represented by a linear combination of the singular fundamental solutions or their partial derivation terms as follows:

$$
\begin{gathered}
u\left(x_{m}\right)=\left\{\begin{array}{l}
\sum_{j=1}^{N} \alpha_{j} G\left(x_{m}, s_{j}\right), \quad x_{m} \in D \backslash \Gamma, \\
\sum_{\substack{j=1 \\
j \neq m}}^{N} \alpha_{j} G\left(x_{m}, s_{j}\right)+\alpha_{m} U_{S}^{j j}, x_{m} \in \Gamma,
\end{array}\right. \\
q\left(x_{m}\right)=\frac{\partial u\left(x_{m}\right)}{\partial \mathbf{n}_{x}}=\left\{\begin{array}{l}
\sum_{j=1}^{N} \alpha_{j} \frac{\partial G\left(x_{m}, s_{j}\right)}{\partial \mathbf{n}_{x}}, \quad x_{m} \in D \backslash \Gamma, \\
\sum_{\substack{j=1 \\
j \neq m}}^{N} \alpha_{j} \frac{\partial G\left(x_{m}, s_{j}\right)}{\partial \mathbf{n}_{x}}+\alpha_{m} Q_{S}^{j j}, x_{m} \in \Gamma,
\end{array}\right.
\end{gathered}
$$

where $N$ is the number of source nodes $s_{j}, \alpha_{j}$ the $j$ th unknown coefficient, $\mathbf{n}_{x}$ the outward normal unit vector on the collocation nodes $x_{m}$, the fundamental solutions $G\left(x_{m}, s_{j}\right)=\left\{\begin{array}{ll}i H_{0}\left(k r_{m j}\right) / 4, & \operatorname{dim}=2 \\ e^{i k r_{m j}} /\left(4 \pi r_{m j}\right), & \operatorname{dim}=3\end{array}, H_{n}^{(1)}\right.$ denotes the $n$th order Hankel function of the first kind, the Euclidean distance $r_{m j}=\left\|x_{m}-s_{j}\right\|_{2}$. If the collocation nodes and source nodes coincide, i.e., $x_{m}=s_{j}$, the well-known singularities in the fundamental solutions are encountered. The concept of the origin intensity factors $U_{S}^{j j}$ and $Q_{S}^{i j}$ is introduced into the SBM for avoiding these singularities. Thanks to the same order of the singularities in 
the Laplace and Helmholtz fundamental solutions [6], the origin intensity factors $U_{S}^{i j}$ and $Q_{S}^{j j}$ can be represented by the following asymptotic expressions:

$$
\begin{gathered}
U_{S}^{j j}=U_{S 0}^{j j}+B, \quad r_{m j} \rightarrow 0, \\
Q_{S}^{i j}=Q_{S 0}^{j j}, \quad r_{m j} \rightarrow 0,
\end{gathered}
$$

where $U_{S 0}^{j j}$ and $Q_{S 0}^{j j}$ are the origin intensity factors of the corresponding Laplace equation [6] $B=\left\{\begin{array}{l}-(\ln (k / 2)+\gamma-i \pi / 2) /(2 \pi), \operatorname{dim}=2 \\ i k /(4 \pi), \\ \operatorname{dim}=3\end{array}\right.$ and $\gamma=0.57721566490153286 \cdots$ is the Euler constant. In this paper, the subtracting and adding-back technique is implemented to derive the formulation of the origin intensity factors $Q_{s 0}^{i j}$ as follows:

$$
Q_{S 0}^{i j}=\frac{1}{L_{m}}-\sum_{\substack{j=1 \\ j \neq m}}^{N} \frac{L_{j}}{L_{m}} \frac{\partial G_{0}\left(x_{m}, s_{j}\right)}{\partial \mathbf{n}_{s}}
$$

where the fundamental solutions of Laplace equation are given by $G_{0}=\left\{\begin{array}{l}-\ln \left(r_{m j}\right) /(2 \pi), \operatorname{dim}=2 \\ 1 /\left(4 \pi r_{m j}\right), \quad \operatorname{dim}=3\end{array}, \mathbf{n}_{s}\right.$ is the outward unit normal vector on the source nodes $s_{j}, L_{j}$ is half-length of the curve $\overline{s_{j-1} s_{j+1}}$ between the source nodes $s_{j-1}$ and $s_{j+1}$ for 2D problems or the infinitesimal area of the source nodes $s_{j}$ for 3D problems as shown in Fig. 1.

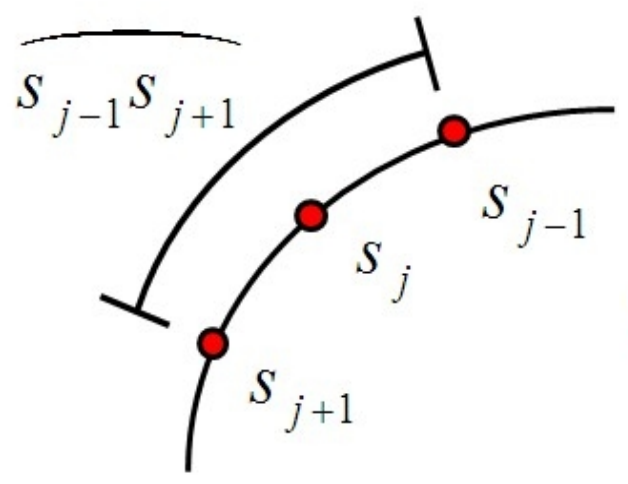

(a)

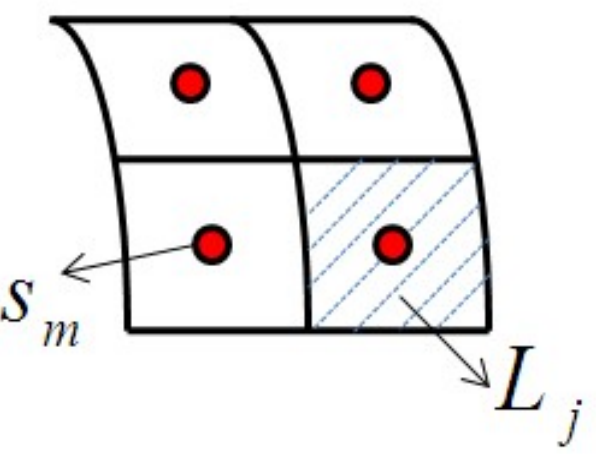

(b)

Figure 1: Schematic configuration. (a) Yhe source points $s_{j}$ and the curve $\widehat{s_{j-1} s_{j+1}}$ for 2D problems; and (b) The source points $s_{j}$ and the corresponding infinitesimal area $L_{j}$ for 3D problems.

For the determination of the origin intensity factors $U_{S 0}^{i j}$, the following empirical formulation is used in $2 \mathrm{D}$ problems [7]: 


$$
U_{S 0}^{j j}=-\frac{1}{2 \pi} \ln \left(\frac{L_{j}}{2 \pi}\right),
$$

and the following formulation is used in 3D problems [11]:

$$
U_{S}^{i j}=-\frac{1}{k L_{m}}\left\{\sum_{\substack{j=1 \\ j \neq m}}^{N}\left[G\left(x_{m}, s_{j}\right) q(s)-\frac{\partial G\left(x_{m}, s_{j}\right)}{\partial n_{s}} u(s)\right] L_{j}-C\left(x_{m}\right) u\left(x_{m}\right)\right\},
$$

where the associated general solution $u(s)$ and its partial derivation terms $q(s)$ can be written as follows:

$$
\begin{gathered}
u(s)=\sum_{d=1}^{3} \sin \left(k\left(s_{j d}-x_{m d}\right)\right) \cdot n\left(x_{m d}\right), \\
q(s)=k \sum_{d=1}^{3} \cos \left(k\left(s_{j d}-x_{m d}\right)\right) \cdot n\left(x_{m d}\right) \cdot n\left(s_{j d}\right),
\end{gathered}
$$

in which $n\left(x_{m d}\right), n\left(s_{j d}\right)$ denotes the Cartesian component of $\mathbf{n}_{x}$ and $\mathbf{n}_{s}$, respectively.

By adapting either eqn (5) or (6) at all the collocation nodes, the SBM formulation for exterior wave propagation problems (eqns (1) and (2)) can be discretized to the following linear system of equations:

$$
\mathbf{A \alpha}=\mathbf{b},
$$

where $\mathbf{A}$ is the SBM resultant matrix, $\boldsymbol{\alpha}$ and $\mathbf{b}$ denote the unknown coefficient vector and the known boundary condition vector, respectively. After determining the vector $\boldsymbol{\alpha}$, the complex-valued amplitude of velocity potential or wave pressure at any discretization nodes inside the domain or on the surface boundary can be evaluated via eqns (5) and (6).

\subsection{Fast Fourier Transform Singular boundary method}

For periodic obstacles, the Fast Fourier Transform can be used to accelerate the SBM matrix computation. Consider two typical periodic soft/rigid obstacles as shown in Fig. 2.

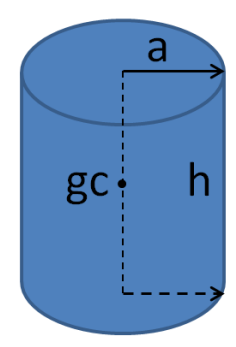

(a)

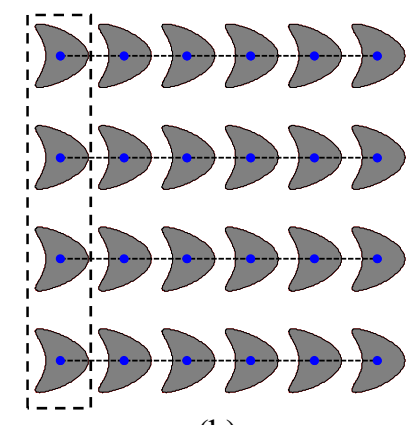

(b)

Figure 2: Schematic configuration of two typical periodic structures. (a) Axisymmetric cylinder structure; (b) Four periodic arrays of scatterers. 
The surface of the kite-shaped obstacle in Figs 2(b) can be represented as

$$
\Gamma=\left\{\left(x_{1}, x_{2}\right): x_{1}=\frac{\cos \varphi+0.65 \cos 2 \varphi-0.65}{6}, x_{2}=0.25 \sin \varphi, 0 \leq \varphi \leq 2 \pi\right\} .
$$

Taking a column of four kite-type scatterers as one obstacle, both the resultant matrices A constructed by the SBM for the typical periodic structures in Fig. 2 are block Toeplitz matrix $\mathbf{T}$ with the form

$$
\mathbf{A}=\mathbf{T}=\left(\begin{array}{cccc}
\mathbf{T}_{0} & \mathbf{T}_{1} & \cdots & \mathbf{T}_{M-1} \\
\mathbf{T}_{-1} & \mathbf{T}_{0} & \ddots & \mathbf{T}_{M-2} \\
\cdots & \ddots & \ddots & \vdots \\
\mathbf{T}_{1-m} & \mathbf{T}_{2-m} & \cdots & \mathbf{T}_{0}
\end{array}\right)
$$

where each $\mathbf{T}_{i}$ denotes an $N \times N$ matrix. The unique block number is $2 M-1$, where $M$ is the number of the scatterers in a row of the periodic structures. As we know, the block Toeplitz matrix can be embedded into a larger block circulant matrix $\mathbf{C}^{\prime}$

$$
\mathbf{C}^{\prime}=\left(\begin{array}{ccccc}
\mathbf{T}_{0} & \mathbf{T}_{1} & \cdots & \mathbf{T}_{-2} & \mathbf{T}_{-1} \\
\mathbf{T}_{-1} & \mathbf{T}_{0} & \mathbf{T}_{1} & \ddots & \mathbf{T}_{-2} \\
\vdots & \mathbf{T}_{-1} & \ddots & \ddots & \vdots \\
\mathbf{T}_{2} & \vdots & \ddots & \ddots & \mathbf{T}_{1} \\
\mathbf{T}_{1} & \mathbf{T}_{2} & \cdots & \mathbf{T}_{-1} & \mathbf{T}_{0}
\end{array}\right)
$$

According to the property of block circulant matrix, only the first block row $\mathbf{R}^{\prime}=\left[\begin{array}{llllllll}\mathbf{T}_{0} & \mathbf{T}_{1} & \cdots & \mathbf{T}_{M-1} & \mathbf{T}_{1-M} & \mathbf{T}_{2-M} & \cdots & \mathbf{T}_{-1}\end{array}\right]$ of $\mathbf{C}^{\prime}$ needs to be computed and stored. Obviously, the computational storage can be reduced to $(2 M-1) / M^{2}$ storage of the full SBM resultant matrix $\mathbf{T}$. Eqn (14) can be rewritten as follows:

$$
\mathbf{C}^{\prime} \boldsymbol{\alpha}^{\prime}=\mathbf{b}^{\prime} \Leftrightarrow\left(\begin{array}{ll}
\mathbf{C}_{11}^{\prime} & \mathbf{C}_{12}^{\prime} \\
\mathbf{C}_{21}^{\prime} & \mathbf{C}_{22}^{\prime}
\end{array}\right)\left(\begin{array}{l}
\boldsymbol{\alpha} \\
\mathbf{0}
\end{array}\right)=\left(\begin{array}{l}
\mathbf{b} \\
\mathbf{d}
\end{array}\right)
$$

where $\mathbf{C}_{11}^{\prime}=\mathbf{T}$ and $\mathbf{d}=\mathbf{C}_{21}^{\prime} \boldsymbol{\alpha}$. It should be mentioned that the linear system of equations (eqn (17)) cannot be solved directly due to the unknown vectors $\boldsymbol{\alpha}$ and $\mathbf{d}$ appeared in both the left-hand and right-hand side vectors $\boldsymbol{\alpha}^{\prime}$ and $\mathbf{b}^{\prime}$. Therefore, the following simple iterative algorithm is implemented to determine unknown vectors $\boldsymbol{\alpha}$ via eqn (17).

i) Substitute the known elements of $\mathbf{b}^{\prime}$ into $\mathbf{b}_{k}^{\prime}$, namely, replace $\mathbf{b}_{k}$ by $\mathbf{b}$ and apply the FFT to compute the following block circulant matrix-vector multiplication:

$$
\mathbf{C}^{\prime-1} \mathbf{b}_{k}^{\prime}=\boldsymbol{\alpha}_{k}^{\prime} \Leftrightarrow \mathbf{C}^{\prime-1}\left(\begin{array}{l}
\mathbf{b}_{k} \\
\mathbf{d}_{k}
\end{array}\right)=\left(\begin{array}{l}
\boldsymbol{\alpha}_{k} \\
\mathbf{e}_{k}
\end{array}\right) \text {. }
$$


ii) Substitute the known elements of $\boldsymbol{\alpha}^{\prime}$ into $\boldsymbol{\alpha}_{k}^{\prime}$, namely, replace $\mathbf{e}_{k}$ by zero vector and apply the FFT to compute the following block circulant matrix-vector multiplication:

$$
\mathbf{C}^{\prime} \boldsymbol{\alpha}_{k}^{\prime}=\mathbf{b}_{k+1}^{\prime} \Leftrightarrow \mathbf{C}^{\prime}\left(\begin{array}{c}
\boldsymbol{\alpha}_{k} \\
\mathbf{0}
\end{array}\right)=\left(\begin{array}{l}
\mathbf{b}_{k+1} \\
\mathbf{d}_{k+1}
\end{array}\right) .
$$

iii) Stop the iteration process if the convergence is achieved with

$$
\left\|\left(\begin{array}{c}
\mathbf{b}_{k+1}-\mathbf{b}_{k} \\
\mathbf{e}_{k}
\end{array}\right)\right\|_{\infty}<\tau_{1},
$$

where $\tau_{1}$ denotes the convergence tolerance. Otherwise restart the loop.

\subsection{Fast multipole singular boundary method}

The fast multipole method (FMM) has been proposed by Greengard and Rokhlin. It consists of computing nearby solutions and far-fields solutions and can be employed to dramatically accelerate the solution procedure of the SBM system of equations (eqn (14)), in which matrix $\mathbf{A}$ is a dense and non-symmetric matrix. The main idea of the fast multipole SBM is to employ iterative solvers (such as GMRES) to solve the matrix system of equations and then employ the FMM to accelerate the matrix-vector multiplication in each iteration step, without forming the matrix A explicitly in general.

In the fast multipole SBM, one can rewrite fundamental solutions as follows:

$$
G\left(x_{m}, s_{j}\right)=\sum_{i} G_{i}^{(1)}\left(x_{m}, z\right) G_{i}^{(2)}\left(s_{j}, z\right) .
$$

Then the node-to-node computations in the conventional SBM are replaced by cell-to-cell interactions using a hierarchical tree structure of cells containing groups of nodes. This is possible by introducing the multipole and local expansions of the kernels and employing certain translations. The roadmap of the FMMSBM can be written as follows:

Step 1 Generate a hierarchical tree partitioning for computational domain;

Step 2 Upward pass. Accumulate multipole expansions for far field interactions through a backward visiting of the tree;

Step 3 Downward pass. Translate multipole moments to local expansions, and construct local expansions through a forward visiting of the tree;

Step 4 Evaluation of all the interactions. Evaluate far-field interactions to particles by local expansions, and evaluate directly near-field interactions.

The details of the FMMSBM formulation and implementation can be found in the literatures [8]. However, for large-scale wave propagation analysis, the FMMSBM still produces a very ill-conditioning resultant matrix, which slows down the convergence rate of matrix-iterative solvers even leads to incorrect results.

\subsection{Dual-level singular boundary method}

To further improve the computable ability of the FMMSBM, the dual level preconditioning technique is introduced to transform the fully populated resultant matrix to a local-supported sparse matrix on the fine level, which may overcome the highly ill-conditioning and 
excessive storage requirement in the FMMSBM solution of large-scale wave propagation. The procedure of the DLFMMSBM (Fig. 3) includes the following steps:

Step 1 (on the coarse level) Evaluation of initial approximate solution on the fine level; Step 2 (on the fine level) Evaluation of initial accurate solution on the fine level;

Step 3 (on the fine level) Assessment of initial average relative error on the fine level; Step 4 (on the fine level) Evaluation of kth accurate residual potential on coarse level; Step 5 (on the coarse level) Evaluation of kth residual potential solution on the coarse level;

Step 6 (on the fine level) Evaluation of $(\mathrm{k}+1)$ th accurate solution on fine level;

Step 7 (on the fine level) Assessment of $(k+1)$ th average relative error on the fine level.

The details of the DLFMMSBM formulation and implementation can be found in the literatures [9].

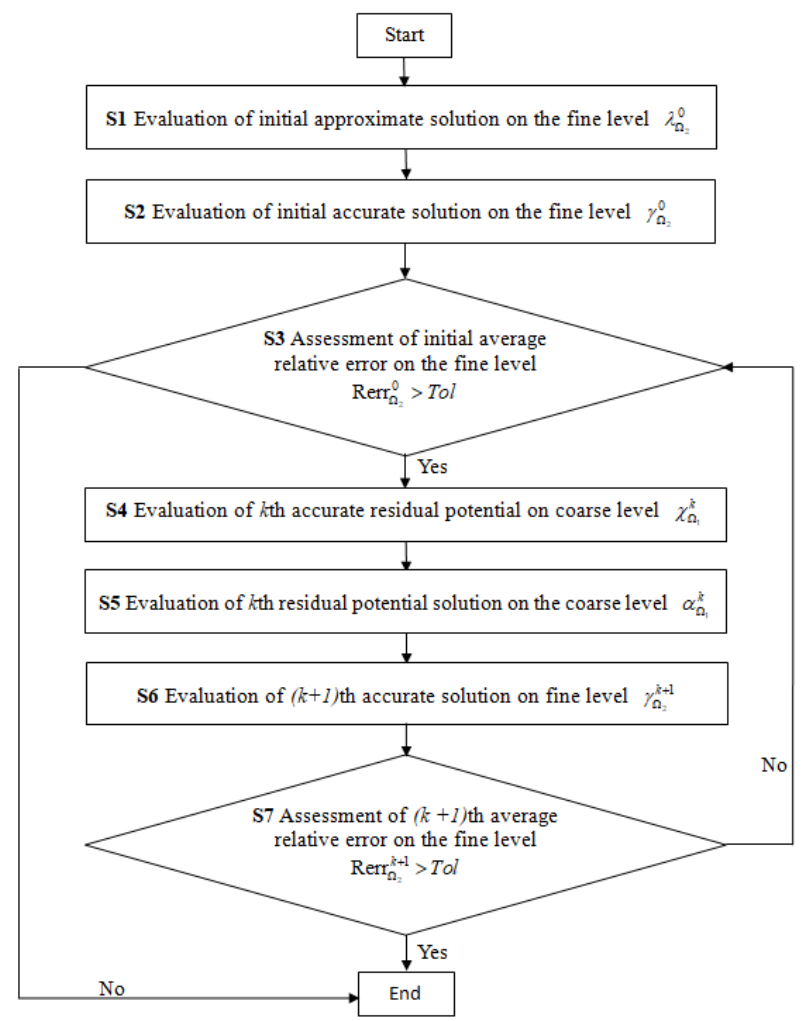

Figure 3: Algorithm diagram of the DLFMMSBM.

\section{NUMERICAL RESULTS AND DISCUSSIONS}

In this section, the efficiency and accuracy of the FFTSBM are first tested on wave propagation by $3 \mathrm{D}$ cylinder structures and four periodic arrays of kite-shaped scatterers. Subsequently, the numerical comparison between DLFMMSBM and FMMSBM is given for wave radiation from a submarine model. The numerical accuracy is measured by the maximum error $\operatorname{Merr}(u)$ defined as 


$$
\operatorname{Merr}(u)=\max _{1 \leq i \leq N T}|u(i)-\bar{u}(i)|,
$$

where $\bar{u}(i)$ and $u(i)$ are the analytical and numerical solutions at $x_{i}$, respectively, and NT is the total number of the test points in the interested domain. The test points $\left\{\mathbf{y}_{j}\right\}$ are placed on a similar geometric surface to the scatterer with its center at the geometric center $g c$ of the scatterer, and the off-distance parameter $o d$ is defines as

$$
o d=\frac{\mathbf{y}_{j}-g c}{\mathbf{y}_{j}^{0}-g c},
$$

where $\left\{\mathbf{y}_{j}^{0}\right\}$ are the points with a uniform angular distribution on the surface of the scatterer. Unless otherwise specified, $N T=200, o d=2, \tau_{1}=10^{-4}$ and $T o l=10^{-4}$ are used in all the following numerical examples on a personal computer with an Intel Core i7 Processor with 16GB RAM.

Example 1: 3D wave radiation by a circular cylinder with soft or rigid boundaries.

First consider the wave propagation by a circular cylinder as shown in Fig. 2(a), where the pressure $(u)$ and the normal velocity $(q)$ on the cylinder surface are induced by a point source of the spherical dilatation wave with the unit intensity located at the geometric center. The analytical solution is presented by

$$
u_{R}\left(r_{g c}, \theta_{g c}, x_{3}\right)=e^{i k r_{g c}} / r_{g c},
$$

where $\left(r_{g c}, \theta_{g c}\right)$ denotes the polar coordinates with the geometric center $g c$ as the origin of the plane $x_{3}=0$. Fig. 4 plots the convergence rate of the FFTSBM with the CPU time versus boundary node number for 3D wave propagation problem with $k a=3 / 16$ and $a=3 h / 16$.

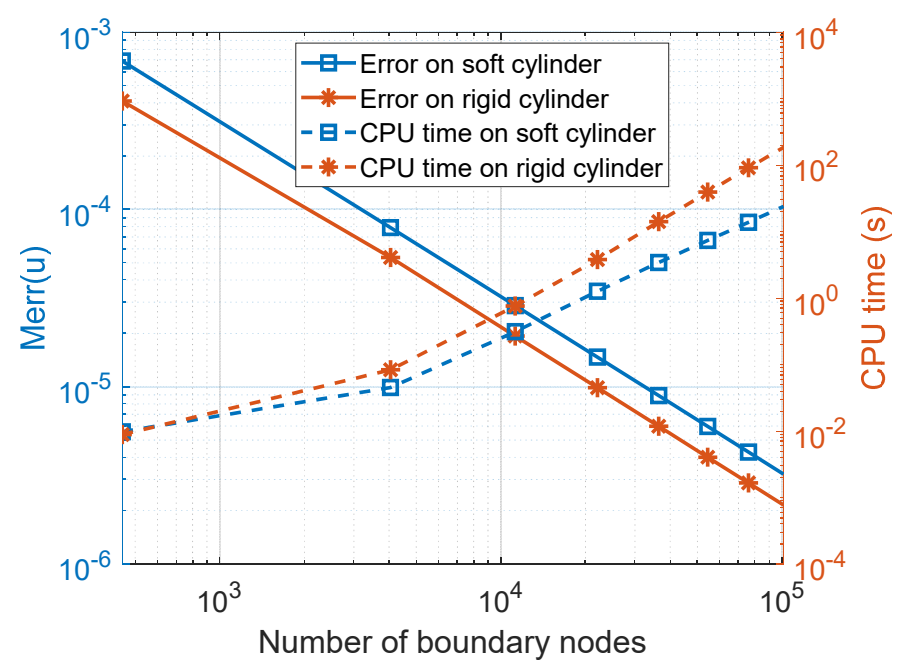

Figure 4: Convergence rate of the FFTSBM with the CPU time versus the boundary node number. 
It can be observed from Fig. 4 that the FFTSBM has a rapid convergence rate with the curve-slope 0.85 , and it only requires about 3 minutes for periodic structure with 1 million boundary nodes.

Example 2: 2D wave scattering by $4 \times 6$ infinite kite-shaped impermeable cylinders.

Next consider the wave interaction with four periodic arrays of infinite kite-shaped impermeable scatterers as shown in Fig. 2(b), which can be simplified to 2D scattering problem of an incident plane wave $e^{i k\left(x_{1} \cos \theta+x_{2} \sin \theta\right)}$ scattered by $4 \times 6$ rigid kite-shaped obstacles. Fig. 5 presents the wave pressure amplitude $|u|$ around this four-periodic-array structure at the incidence wave angle $\theta=\pi / 4$ and the dimensionless wavenumber $k a=\pi / 2$ by using 50 boundary nodes on each kite-shaped obstacle surface.

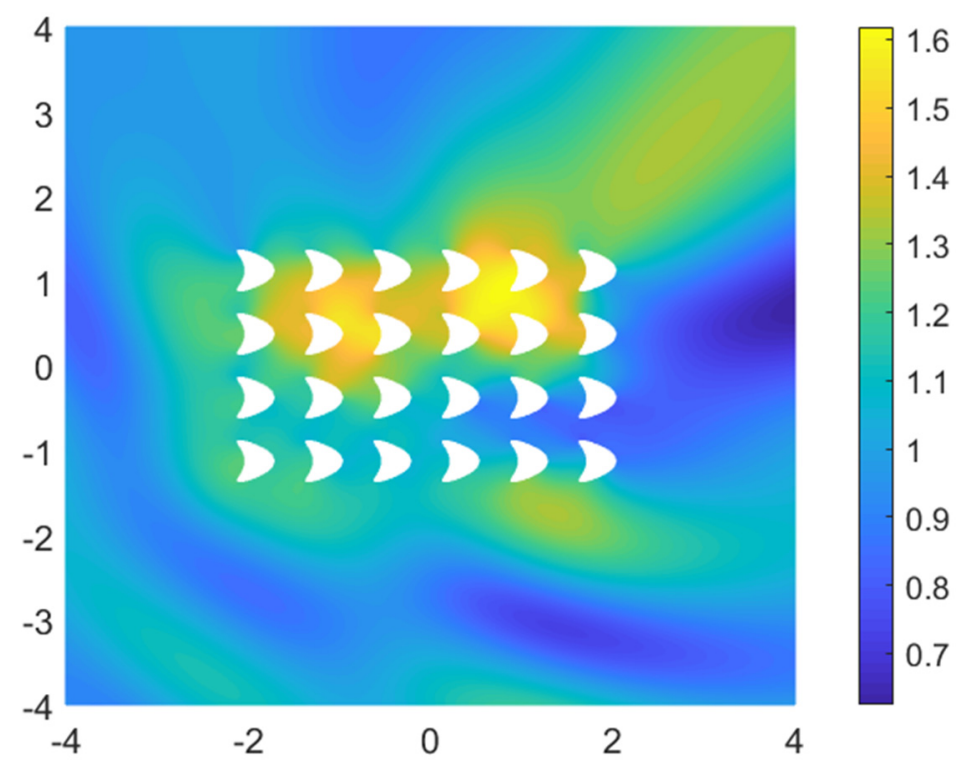

Figure 5: Wave pressure amplitude $|u|$ for the $4 \times 6$ kite-shaped cylinder array at the incidence wave angle $\theta=\pi / 4$ and the dimensionless wavenumber $k a=\pi / 2$ by using FFTSBM.

Example 3: 3D wave radiation by a submarine model (Fig. 6)

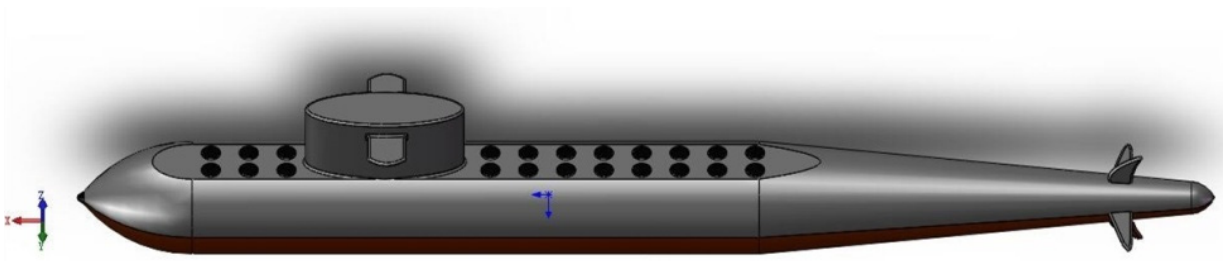

Figure 6: A submarine model. 
We consider the wave propagation by a submarine model with a size of $120 \mathrm{~m} \times 12 \mathrm{~m} \times 12 \mathrm{~m}$ as shown in Fig. 6, where acoustic wave speed is set as $1500 \mathrm{~m} / \mathrm{s}$. The analytical solution is given by

$$
u_{R}\left(x_{1}, x_{2}, x_{3}\right)=x_{3} e^{i k r}\left(1+\frac{i}{k r}\right) / r^{2},
$$

The test points are uniformly distributed on the surface of sphere with radius $100 \mathrm{~m}$. Table 1 lists the numerical comparison between FMMSBM and DLFMMSBM in Example 3 at two certain wave frequencies $(f=87$ and $217 \mathrm{~Hz})$. It can be found from Table 1 that the FMM is introduced to allow the SBM to solve the large-scale wave propagation by complicated-geometry structure with more than 1 million boundary nodes. Moreover, the DLFMMSBM provides more accurate solutions with less CPU time and fewer boundary node requirement than those of the FMMSBM.

Table 1: Numerical comparison between FMMSBM and DLFMMSBM in Example 3 at two certain wave frequencies $(f=87$ and $217 \mathrm{~Hz}$ ).

\begin{tabular}{|l|c|c|c|c|}
\hline & \multicolumn{2}{|c|}{ DLFMM+SBM } & \multicolumn{2}{c|}{ FMM+SBM [8] } \\
\hline$k d(d=120 m)$ & 44.4 & 110.9 & 44.4 & 110.9 \\
\hline$f(H z)$ & 87 & 217 & 87 & 217 \\
\hline Coarse-level nodes & 5053 & 8019 & $/$ & $/$ \\
\hline Fine-level nodes & 65,058 & 100,096 & 63,488 & $1,030,876$ \\
\hline Rerr $($ Tol=1e-4) & $1.93 \mathrm{E}-4$ & $5.04 \mathrm{E}-4$ & $1.13 \mathrm{E}-3$ & $2.30 \mathrm{E}-2$ \\
\hline CPU time $(s)$ & $6.76 \mathrm{E}+1$ & $2.09 \mathrm{E}+2$ & $7.57 \mathrm{E}+2$ & $9.24 \mathrm{E}+3$ \\
\hline
\end{tabular}

\section{CONCLUSIONS}

This paper presents the singular boundary method (SBM) in conjunction with the fast dense matrix solvers for large-scale wave propagation analysis. The Fast Fourier Transform (FFT) is introduced to the proposed SBM for solving periodic structure with 1 million boundary nodes about 3 minutes. The fast multipole method allows the SBM to solve the large-scale wave propagation by complicated-geometry structure with more than 1 million boundary nodes about 150 minutes. Furthermore, the dual-level preconditioning scheme enables the FMMSBM only to place $2-3$ coarse-level nodes to obtain the acceptable numerical results. This node requirement almost approaches the minimum requirement specified by the Shannon's sampling theorem. It is worth noting that the proposed DLFMMSBM for large-scale wave propagation analysis with complicated-geometry structure at high wavenumber is still under intense study.

\section{ACKNOWLEDGEMENTS}

The work described in this paper was supported by the National Science Fund of China (Grant No. 11772119, 11572111), Alexander von Humboldt Research Fellowship (ID: 1195938) and Qing Lan Project. 


\section{REFERENCES}

[1] Ciskowski, R.D. \& Brebbia, C.A. (eds), Boundary Element Methods in Acoustics, Springer-Verlag: Berlin and New York, 1991.

[2] Fairweather, G., Karageorghis, A. \& Martin, P.A., The method of fundamental solutions for scattering and radiation problems. Engineering Analysis with Boundary Elements, 27(7), pp. 759-769, 2003.

[3] Chen, W., Singular boundary method: a novel, simple, meshfree, boundary collocation numerical method. Acta Mechanica Solida Sinica (Chinese), 30, pp. 592-599, 2009.

[4] Fu, Z.J. \& Chen, W., A novel boundary meshless method for radiation and scattering problems. Advances in Boundary Element Techniques XI, pp. 83-90, 2010.

[5] Fu, Z.J., Chen, W., Chen, J.T. \& Qu, W.Z., Singular boundary method: three regularization approaches and exterior wave applications. Computer Modeling in Engineering \& Sciences, pp. 1-26, 2014.

[6] Fu, Z.J., Chen, W. \& Gu, Y., Burton-Miller-type singular boundary method for acoustic radiation and scattering. Journal of Sound and Vibration, 333(16), pp. 37763793, 2014.

[7] Fu, Z., Chen, W., Wen, P. \& Zhang, C., Singular boundary method for wave propagation analysis in periodic structures. Journal of Sound and Vibration, 425, pp. 170-188, 2018.

[8] Qu, W., Chen, W. \& Zheng, C., Diagonal form fast multipole singular boundary method applied to the solution of high-frequency acoustic radiation and scattering. International Journal for Numerical Methods in Engineering, 111(9), pp. 803-815, 2017.

[9] Li, J., Chen, W. \& Fu, Z., A modified dual-level algorithm for large-scale threedimensional Laplace and Helmholtz equation. Computational Mechanics, 62(4), pp. 893-907, 2018.

[10] Li, J., Chen, W., Qin, Q.H. \& Fu, Z., A modified multilevel algorithm for large-scale scientific and engineering computing. Computers \& Mathematics with Applications, 77(8), pp. 2061-2076, 2019.

[11] Li, J., Chen, W., Fu, Z., \& Qin, Q.H., A regularized approach evaluating the nearboundary and boundary solutions for three-dimensional Helmholtz equation with wideband wavenumbers. Applied Mathematics Letters, 91, pp. 55-60, 2019. 